\title{
Brain Natriuretic Peptide as an Additional Metric for Determining Operative Candidates among Veterans with Aortic Stenosis
}

\author{
Matthew Skancke1, Jacob Lambdin', Richard Amdur³, Jason Morrisette², Michael Greenberg2, \\ Gregory Trachiotis ${ }^{1,3}$
}

${ }^{1}$ Division of Cardiothoracic Surgery, Veterans Affairs Medical Center, Washington DC, USA

${ }^{2}$ Division of Cardiology, Veterans Affairs Medical Center, Washington DC, USA

${ }^{3}$ Division of Cardiothoracic Surgery, the George Washington University, Washington DC, USA

Email: Gregory.Trachiotis@va.gov

How to cite this paper: Skancke, M., Lambdin, J., Amdur, R., Morrisette, J., Greenberg, M. and Trachiotis, G. (2017) Brain Natriuretic Peptide as an Additional Metric for Determining Operative Candidates among Veterans with Aortic Stenosis. World Journal of Cardiovascular Surgery, 7, 111-118.

https://doi.org/10.4236/wjcs.2017.79013

Received: August 10, 2017

Accepted: September 4, 2017

Published: September 7, 2017

Copyright $\odot 2017$ by authors and Scientific Research Publishing Inc. This work is licensed under the Creative Commons Attribution International License (CC BY 4.0).

http://creativecommons.org/licenses/by/4.0/

\begin{abstract}
Objective: As the veteran population ages, the incidence of clinically significant aortic stenosis (AS) is increasing and aortic stenosis has become a veterans' health issue. This analysis focused on using serum Brain Natriuretic Peptide (BNP) levels as an adjunct to aid decision making for early aortic valve replacement (AVR) in veterans with severe AS to reduce hospital admission rates. Methods: We retrospectively reviewed the charts of patients referred to the heart valve clinic at the Washington DC, Veterans Affairs Medical Center (VAMC) Heart Center between 2004 and 2015 who were diagnosed with severe AS. We identified veterans who had a BNP drawn in addition to traditional echocardiography during their diagnostic workup. This cohort was then stratified based on their serum BNP levels and whether they received medical therapy or aortic valve replacement. The primary endpoint of interest was admission to a VAMC for valvular heart failure. Results: Univariate analysis of BNP quartile and operative status showed a reduction in number of admissions for veterans who underwent AVR with BNP between 101 and 300 (0.64 vs. $3.71, \mathrm{p}=0.054)$ and 301 and 1000 ( 1.36 vs. $4.0, \mathrm{p}=0.003)$ compared to veterans treated medically. There was no difference in number of admissions for veterans with BNP lower than $100(\mathrm{p}=0.455)$ or higher than $1000(\mathrm{p}=$ 0.659). Conclusion: BNP may be a useful adjunct for selecting patients with AS for earlier AVR leading to lower rates of hospital admissions in the veteran population. Continued analysis in a larger cohort will be needed to further validate the utility of BNP stratification as a diagnostic tool to risk stratify patients with AS in a heart valve clinic.
\end{abstract}




\section{Keywords}

BNP, Aortic Stenosis, Valve Replacement

\section{Introduction}

B-type natriuretic peptide (BNP) is a 32-amino-acid molecule which acts as a biomarker reporting damage to cardiac myocytes. BNP is released from damaged myocardium in the setting of ventricular distention or tension [1], or myocardial ischemia [2] and is correlated to adverse outcomes following both cardiac [3] and non-cardiac surgeries [4]. Expanding on our previous work that evaluated BNP as a variable in preoperative surgical risk stratification, this analysis compares aortic valve replacement (AVR) versus medical therapy for patients with aortic stenosis (AS) based on BNP grouping.

Current recommended from the American Heart Association and the American College of Cardiology (AHA/ACC) suggest AVR be considered for patients with: symptomatic severe aortic stenosis (AS) with high-gradient (aortic valve area $\leq 1 \mathrm{~cm}^{2}$ with aortic $\operatorname{Vmax}>4 \mathrm{~m} / \mathrm{s}$ or mean $\Delta \mathrm{P}>40 \mathrm{~mm} \mathrm{Hg}$ ), severe AS with LVEF of < 50\% [5] [6] [7], severe AS when undergoing other cardiac surgery, or asymptomatic patients with severe AS and rapid interval progression of stenosis (defined as an increase in Aortic Vmax by $\geq 0.3 \mathrm{~m} / \mathrm{s}$ over the course of $\leq 2$ years) [2]. The AHA/ACC also recommends that AVR should be considered for asymptomatic patients with critical AS (defined as aortic $V \max \geq 4 \mathrm{~m} / \mathrm{s}$ ) and low surgical risk, patients with severe AS with decreased exercise tolerance or exercised induced drop in blood pressure [2]. However, despite the detailed structural guidelines for patients with AS, clinical judgement is still heavily relied on identifying patients who would benefit from AVR, and best therapy decided by a heart valve team.

This analysis focused on the novel use of BNP as an additional biologic diagnostic metric in patients with severe AS. We hypothesized that increased BNP levels would correlate with increased disease related hospital admissions in the medically and surgically managed AS groups. However, given the structural nature of AS, early AVR would help reduce hospital admissions when compared to veterans with AS managed medically. Establishing the utility of BNP for selecting those who would benefit from early AVR could potentially reduce healthcare costs and decrease morbidity related to readmission to the hospital for AS and heart failure related to AS.

\section{Methods}

An institutional review board-approved retrospective review was performed for Veterans treated for AS at the Heart Valve Clinic (HVC) and by the Heart Valve Team (HVT) [8] (Noninvasive imaging Cardiology, Structural Heart Cardiology, Cardiothoracic Surgeon, Heart Valve Coordinator, Physi- 
cian Assistants) at a single Veterans Affairs Medical Center (VAMC) between 2004 to 2015 by a single cardiac surgeon. Patient demographic and clinical data (Age, BMI, Metabolic Equivalents, Coronary Artery Disease, Hypertension, Hyperlipidemia, Diabetes, Chronic Obstructive Pulmonary Disease, End Stage Renal Disease, Atrial Fibrillation, Congestive Heart Failure) was acquired using the Veterans Information Systems and Technology Architecture (VISTA) and the Computerized Patient Record System (CPRS). VISTA and CPRS are two components of the national electronic health record utilized by the VA to provide and track patient care. Patients included had a detailed echocardiographic analysis (Aortic Valve Area, Max Aortic Valve Gradient, Mean Aortic Valve Gradient, Aortic Jet Velocity, Ejection Fraction) and a serum BNP as part of their first HVC appointment. The decision for BNP grouping was based on our prior analysis of the effect of BNP on postoperative morbidity and mortality following cardiac surgery [3]. Early aortic valve replacement was the intervention for this analysis compared to interval monitoring and a "watch and wait" strategy. The primary endpoint of interest was the number of admissions to a VAMC following the initial HVC appointment. Admissions to a VAMC were included if the primary diagnosis code linked with the admission involved aortic stenosis or valvular heart failure related to aortic stenosis.

Data acquired from HVT records were examined for outliers and non-normality of distribution. Records with outlier or missing data in critical fields were not included in this analysis. Preoperative demographics were evaluated using t-testing for continuous variables and $\mathrm{x} 2$ for nominal variables. Univariate analysis compared BNQ quartile and medical versus surgical treatment with respect to the primary outcome of interest, the number of hospital admissions following initial valve clinic appointment. IBM SPSS version 24 statistical software was used for the analysis with $\mathrm{p}<0.05$ holding significance.

\section{Results}

Our inclusion criteria identified 75 male veterans who were treated at the cardiac valve clinic who also had echocardiographic data and a serum BNP as components of their initial HVC workup. Veteran grouping based on serum BNP was equal between medical and surgical arms (Table 1). Those in the medical group were slightly older (76 vs. $71, \mathrm{p}=0.025$ ), had a higher rate of coronary disease ( $44 \%$ vs. $22 \%, \mathrm{p}=0.041$ ), had a higher rate of chronic obstructive pulmonary disease $(24 \%$ vs. $7 \%, \mathrm{p}=0.049)$ and had a higher rate of congestive heart failure $(24 \%$ vs. $5 \%, p=0.018)$. Veterans in the surgical group had symptomatic aortic stenosis, and a higher incidence of diabetes $(54 \%$ vs. $32 \%, p=0.066)$. Other reported preoperative factors were similar between groups (Table 2). Echocardiographic imaging studies (Table 3 ) indicated that veterans in the surgical group had more advanced aortic stenosis compared to the medical group: aortic valve 
Table 1. Serum BNP quartiles of enrolled veterans treated by the heart valve clinic.

\begin{tabular}{cccc}
\hline Serum BNP (\%, $\mathbf{n})$ & Surgical $(\mathbf{n}=\mathbf{4 1})$ & Medical (n = 34) & P \\
\hline$<100$ & $52 \%, 13$ & $48 \%, 12$ & \\
$101-300$ & $63 \%, 12$ & $37 \%, 7$ & 0.204 \\
$301-1000$ & $67 \%, 12$ & $33 \%, 6$ & \\
$>1000$ & $31 \%, 4$ & $69 \%, 9$ & \\
\hline
\end{tabular}

Serum BNP quartiles for veterans treated in the HVC between 2004 and 2015. Quartile selection based on previous research (Endicott et al., 2016) which showed a relationship between higher levels of preoperative serum BNP and increased morbidity and long-term mortality following cardiac surgery at a VAMC.

Table 2. Demographics of enrolled veterans treated by the heart valve clinic.

\begin{tabular}{cccc}
\hline Factor (Average, SEM, $\mathbf{n})$ & Surgical $(\mathbf{n}=41)$ & Medical (n = 34) & P \\
\hline Age (Years) & $71.41,1.52$ & $76.62,1.70$ & 0.025 \\
Body Mass Index $\left(\mathrm{kg} / \mathrm{m}^{2}\right)$ & $32.2,1.91$ & $29.19,0.93$ & 0.179 \\
Metabolic Equivalents & $5.36,0.97$ & $6.34,0.70$ & 0.406 \\
Coronary Artery Disease & $22 \%, 6.5 \%, 9$ & $44 \%, 8.6 \%, 15$ & 0.041 \\
Hypertension & $85 \%, 5.6 \%, 35$ & $71 \%, 7.9 \%, 24$ & 0.123 \\
Hyperlipidemia & $54 \%, 7.9 \%, 22$ & $47 \%, 8.7 \%, 16$ & 0.575 \\
Diabetes & $54 \%, 7.9 \%, 22$ & $32 \%, 8.1 \%, 11$ & 0.066 \\
COPD & $7 \%, 4.1 \%, 3$ & $24 \%, 7.4 \%, 8$ & 0.049 \\
End Stage Renal Disease & $17 \%, 5.9 \%, 7$ & $15 \%, 6.2 \%, 5$ & 0.784 \\
Atrial Fibrillation & $15 \%, 5.6 \%, 6$ & $18 \%, 6.6 \%, 6$ & 0.727 \\
Congestive Heart Failure & $5 \%, 3.4 \%, 2$ & $24 \%, 7.4 \%, 8$ & 0.018 \\
\hline
\end{tabular}

Preoperative factors for veterans with previously diagnosed aortic stenosis treated by the HVC at the VAMC; continuous variables are reported as means and categorical variables are reported in percentages. SEM refers to the standard error of the means for each value. ${ }^{*}$ Chronic Obstructive Pulmonary Disease (COPD).

Table 3. Echocardiographic metrics of enrolled veterans treated by the heart valve clinic.

\begin{tabular}{cccc}
\hline Metric (Average, SEM) & Surgical $(\mathrm{n}=41)$ & Medical $(\mathrm{N}=34)$ & $\mathbf{P}$ \\
\hline Aortic Valve Area $\left(\mathrm{cm}^{2}\right)$ & $0.79,0.031$ & $1.05,0.062$ & $<0.001$ \\
Max Gradient $(\mathrm{mmHg})$ & $63.12,2.84$ & $55.18,3.2$ & 0.069 \\
Mean Gradient $(\mathrm{mmHg})$ & $38.37,1.86$ & $32.86,1.91$ & 0.046 \\
Aortic Jet Velocity $(\mathrm{m} / \mathrm{s})$ & $3.93,0.12$ & $3.52,0.17$ & 0.046 \\
Ejection Fraction $(\%)$ & $50.25 \%, 1.58 \%$ & $51.5 \%, 1.53 \%$ & 0.577 \\
\hline
\end{tabular}

Echocardiographic metrics for veterans with aortic stenosis treated medically and surgically by the HVC. Continuous variables reported as means. Categorical variables reported in percentages. SEM refers to the standard error of the means for each value.

area (0.79 vs. $1.05 \mathrm{~cm} 2, \mathrm{p}<0.001)$, max gradient (63.12 vs. $55.18, \mathrm{p}=0.069)$ $\mathrm{mmHg}$, mean gradient ( 38.37 vs. $32.86, \mathrm{p}=0.046) \mathrm{mmHg}$, and jet velocity ( 3.93 vs. $3.52, \mathrm{p}=0.046) \mathrm{m} / \mathrm{s}$. The average ejection fraction was greater than $50 \%$ in 
both groups $(\mathrm{p}=0.577)($ Table 3$)$.

Univariate analysis was utilized to compare the number of admissions for aortic valve pathology following the initial HVC in veterans who were treated medical versus those who underwent AVR (Table 4). There was no difference in the number of admissions following AVR for veterans with BNP $<100(0.33$ vs. $0.17, \mathrm{p}=0.455)$ or in veterans with $\mathrm{BNP}>1001(1.75$ vs. $1.25, \mathrm{p}=0.659)$. There was a significant reduction in hospital admissions following AVR for veterans in the 101 to 300 ( 3.71 vs. $0.64, \mathrm{p}=0.054$ ) and 301 to 1000 (4.0 vs. $1.36, \mathrm{p}=0.003)$.

\section{Discussion}

This analysis adds credence to a strategy that utilizes BNP as a biologic diagnostic metric to identify veterans with severe AS who would benefit from early AVR versus continued medical management. While AVR is indicated for the treatment of symptomatic severe AS, the treatment asymptomatic severe AS remains controversial. Biologic objective diagnostic metrics could supplement the existing guidelines put forth by the ACC/AHA regarding the treatment of AS by helping further stratify candidates for surgical valve replacement. We believe that BNP could be considered for inclusion into the next iteration of the ACC/ AHA guidelines for the treatment of AS because of its nature as a harbinger for loss of ventricular compensation [9] and proven track record as a prognostic indicator for negative outcomes following medical or surgical management.

Historically, changes in structural metrics were considered the gold standard for progression of aortic stenosis, however, as study into cardiac physiology has progressed biologic markers have become readily available and may provide a more predictive measure of disease progression [10] [11]. Amongst the available biomarkers, BNP (normal level less than 100) and NT-proBNP (normal level less than 1000) [12] have been recognized as a strong prognosticator for morbidity and mortality associated with AS and AVR [3] [13] [14] [15] [16]. Between these two natriuretic peptides, NT-proBNP is considered by some to be a more sensitive marker for heart failure [17] potentially in part because of its longer half-life

Table 4. Univariate analysis of BNP quartile and early valve replacement on readmission.

\begin{tabular}{cccc}
\hline BNP (Average \#, SEM, $\mathbf{n})$ & Surgical $(\mathbf{n}=38)$ & Medical $(\mathbf{n}=33)$ & $\mathbf{P}$ \\
\hline$<100$ & $0.17,0.11,12$ & $0.33,0.19,12$ & 0.455 \\
$101-300$ & $0.64,0.31,11$ & $3.71,1.82,7$ & 0.054 \\
$301-1000$ & $1.36,0.43,11$ & $4.0,0.632,6$ & 0.003 \\
$>1000$ & $1.25,0.629,4$ & $1.75,0.70,8$ & 0.659 \\
\hline
\end{tabular}

Univariate analysis between BNP quartile and operative vs. medical treatment on number of re-admission to a VAMC for aortic stenosis or heart failure related to aortic stenosis following the initial heart valve clinic appointment. Note the significant decrease in hospital admission for patients undergoing early surgical therapy with BNP values between 101 and 300 trending to significance and the significant decrease in admissions in the 301 to 1000 group. No significant reduction in admission was seen with lower or exceedingly high serum BNP values. 
compared to BNP (25 to 75 minutes vs. 20 minutes for BNP) [18]. NT-proBNP has also been validated as a prognostic marker for adverse outcomes in patients with heart failure [19]. Given the time frame of our study, BNP was chosen as the metric for stratification however we are currently in the process of migrating to NT-proBNP as well to help guide treatment of severe AS in our veterans.

These results confirm our initial hypothesis that veterans with severe AS and BNP between 101 - 300 or 301 - 1000 treated with early AVR have fewer hospital admissions for aortic stenosis or related heart failure following their initial HVC evaluation compared to those treated medically. While the decrease in hospital admissions is statistically significant, we recognize that there are differences in the medical and surgical cohort that may influence these results. Patients in the medical cohort were older and had higher rates of CHF disease, COPD and diabetes. Conversely, patients in the surgical cohort had higher rates of CAD, and had symptomatic AS. Given the retrospective nature of this analysis, it is difficult to assess the impact of these comorbidities on our primary outcome. It is also important to note that our cohort only included male veterans treated by the HVT. Some of the bias injected by the differences in cohorts is mitigated by only including hospital admissions related to aortic stenosis or heart failure related to aortic stenosis.

Another important distinction between the medical and surgical cohorts is the difference in echocardiographic metrics. Aortic valve area, max gradient, mean gradient and aortic jet velocity were more severe in the surgical cohort. As a result, there was likely more cardiac remodeling in the surgical cohort prior to AVR which may not have resolved after the pathologic aortic valve was replaced.

Despite these limitations, this analysis provides a stepping stone for subsequent studies focused on the additive benefits of BNP as a diagnostic biologic metric to aid the management of severe AS managed by a HVT [8]. In select patient with asymptomatic severe AS or functionally limited moderate to severe AS who also have an elevated BNP or NT-proBNP, this analysis suggests a reduction in future hospital admissions for AS related heart failure with early AVR versus non-operative management. Moreover, in clinical scenarios where functional testing is not reliable in gauging symptomatic severe AS, BNP or NT-proBNP may help guide therapy for either early aortic valve replacement. Based on this analysis, we have changed our practice to offer early AVR for veterans with severe AS and an elevated BNP and recommend that other surgeons strongly consider early AVR under similar circumstances to help reduce hospital admissions for heart failure in this population.

\section{Conclusion}

Veterans with aortic stenosis and a BNP between 100 and 1000 may have fewer admissions to the hospital for aortic valve pathology when treated with early aortic valve replacement compared to similar veterans managed non-operatively. A continued analysis in a larger cohort would be beneficial to further validate 
the utility of BNP as a biologic diagnostic tool to risk stratify patients with asymptomatic severe aortic stenosis followed by a Heart Valve Team.

\section{References}

[1] Levin, E.R., Gardner, D.G. and Samson, W.K. (1998) Natriuretic Peptides. The New England Journal of Medicine, 339, 321-328. https://doi.org/10.1056/NEJM199807303390507

[2] Nishimura, R.A., Otto, C.M., Bonow, R.O., et al. (2014) 2014 AHA/ACC Guideline for the Management of Patients With Valvular Heart Disease: Executive Summary: A Report of the American College of Cardiology/American Heart Association Task Force on Practice Guidelines. Circulation, 129, 2440-2492. https://doi.org/10.1161/CIR.0000000000000029

[3] Endicott, K.M., Amdur, R.L., Greenberg, M.D. and Trachioti,s G.D. B-Type Natriuretic Peptide Predicts Morbidity and Long-Term Mortality in Coronary Artery Bypass Grafting and Valve Surgery. Innovations (Phila), 11, 439-443.

[4] Goetze, J.P., Christoffersen, C., Perko, M., et al. (2003) Increased Cardiac BNP Expression Associated with Myocardial Ischemia. The FASEB Journal, 17, 1105-1107.

[5] Connolly, H.M., Oh, J.K., Orszulak, T.A., et al. (1997) Aortic Valve Replacement for Aortic Stenosis with Severe Left Ventricular Dysfunction. Prognostic Indicators. Circulation, 95, 2395-400. https://doi.org/10.1161/01.CIR.95.10.2395

[6] Pereira, J.J., Lauer, M.S., Bashir, M., et al. (2002) Survival after Aortic Valve Replacement for Severe Aortic Stenosis with Low Transvalvular Gradients and Severe Left Ventricular Dysfunction. Journal of the American College of Cardiology, 39, 1356-1363. https://doi.org/10.1016/S0735-1097(02)01759-X

[7] Pai, R.G., Varadarajan, P. and Razzouk, A. (2008) Survival Benefit of Aortic Valve Replacement in Patients with Severe Aortic Stenosis with Low Ejection Fraction and Low Gradient with Normal Ejection Fraction. The Annals of Thoracic Surgery, 86, 1781-1789.https://doi.org/10.1016/j.athoracsur.2008.08.008

[8] Endicott, K., Lambdin, J., Morrisette, J., et al. (2017) Heart Valve Clinic: A Model for Treatment of Structural Heart Disease. World Journal of Cardiovascular Surgery, 7, 1-9. https://doi.org/10.4236/wjcs.2017.71001

[9] Henri, C., Dulgheru, R., Magne, J., et al. (2016) Impact of Serial B-Type Natriuretic Peptide Changes for Predicting Outcome in Asymptomatic Patients With Aortic Stenosis. The Canadian Journal of Cardiology, 32, 183-189.

https://doi.org/10.1016/j.cjca.2015.06.007

[10] Vanderheyden, M., Goethals, M., Verstreken, S., et al. (2004) Wall Stress Modulates Brain Natriuretic Peptide Production in Pressure Overload Cardiomyopathy. Journal of the American College of Cardiology, 44, 2349-2354.

https://doi.org/10.1016/j.jacc.2004.09.038

[11] Clavel, M.-A., Malouf, J., Michelena, H.I., et al. (2014) B-Type Natriuretic Peptide Clinical Activation in Aortic Stenosis: Impact on Long-Term Survival. Journal of the American College of Cardiology, 63, 2016-2025. https://doi.org/10.1016/j.jacc.2014.02.581

[12] Waldo, S.W., Beede, J., Isakson, S., et al. (2008) Pro-B-Type Natriuretic Peptide Levels in Acute Decompensated Heart Failure. Journal of the American College of Cardiology, 51, 1874-1882. https://doi.org/10.1016/j.jacc.2007.12.051

[13] Goodman, A., Kusunose, K., Popovic, Z.B., et al. (2016) Synergistic Utility of Brain Natriuretic Peptide and Left Ventricular Strain in Patients with Significant Aortic 
Stenosis. Journal of the American Heart Association, 5, Article ID: e002561.

[14] Abramowitz, Y., Chakravarty, T., Jilaihawi, H., et al. (2015) Impact of Preprocedural B-Type Natriuretic Peptide Levels on the Outcomes after Transcatheter Aortic Valve Implantation. American Journal of Cardiology, 116, 1904-1909. https://doi.org/10.1016/j.amjcard.2015.09.031

[15] McKie, P.M. and Burnett, J.C. (2016) NT-proBNP: The Gold Standard Biomarker in Heart Failure. Journal of the American College of Cardiology, 68, 2437-2439. https://doi.org/10.1016/j.jacc.2016.10.001

[16] Raymond, I., Groenning, B.A., Hildebrandt, P.R., et al. (2003) The Influence of Age, Sex and Other Variables on the Plasma Level of N-Terminal Pro Brain Natriuretic Peptide in a Large Sample of the General Population. Heart, 89, 745-751. https://doi.org/10.1136/heart.89.7.745

[17] Fonseca, C., Sarmento, P.M., Minez, A., et al. (2014) Comparative Value of BNP and NT-proBNP in Diagnosis of Heart Failure. Revista Portuguesa de Cardiologia, 23, 979-991.

[18] Mair, J. (2008) Biochemistry of B-Type Natriuretic Peptide-Where Are We Now? Clinical Chemistry and Laboratory Medicine, 46, 1507-1514.

https://doi.org/10.1515/CCLM.2008.295

[19] Hartmann, F., Packer, M., Coats, A.J.S., et al. (2004) Prognostic Impact of Plasma N-Terminal Pro-Brain Natriuretic Peptide in Severe Chronic Congestive Heart Failure: A Substudy of the Carvedilol Prospective Randomized Cumulative Survival (COPERNICUS) Trial. Circulation, 110, 1780-1786.

https://doi.org/10.1161/01.CIR.0000143059.68996.A7

Submit or recommend next manuscript to SCIRP and we will provide best service for you:

Accepting pre-submission inquiries through Email, Facebook, LinkedIn, Twitter, etc. A wide selection of journals (inclusive of 9 subjects, more than 200 journals)

Providing 24-hour high-quality service

User-friendly online submission system

Fair and swift peer-review system

Efficient typesetting and proofreading procedure

Display of the result of downloads and visits, as well as the number of cited articles

Maximum dissemination of your research work

Submit your manuscript at: http://papersubmission.scirp.org/

Or contact wjcs@scirp.org 